\title{
THE TERRORIST STILL LURKS IN THE HOSPITAL- PREVALENCE OF MRSA
}

\author{
Indrani Bhattacharyya ${ }^{1}$, Debarati Banerjee ${ }^{2}$
}

${ }^{1}$ Associate Professor, Department of Microbiology, CNMC, Kolkata, West Bengal, India.

2Demonstrator, Department of Microbiology, CNMC, Kolkata, West Bengal, India.

\section{BACKGROUND}

ABSTRACT

MRSA poses a very serious problem producing difficult to treat infections in the community as well as in health facilities. Out of these, Health Care associated MRSA infections have the additional problem of being multidrug resistant. This fact led us to conduct this study in a tertiary care hospital in Kolkata.

\section{MATERIALS AND METHODS}

Samples of pus were collected from all the wards of the hospital. Samples were received within 30 minutes of their collection. The usual protocol of Gram stain, culture, biochemical tests and antibiotic sensitivity was followed. Phenotypic method using $30 \mu \mathrm{g}$ cefoxitin disc was followed for identifying MRSA according to CLSI guidelines. The results were tabulated, and prevalence of MRSA was calculated.

\section{RESULTS}

Out of 102 Staphylococcus aureus samples 35.3\% were found to be methicillin resistant. This prevalence of MRSA is found to be at par with a study done by Sangram S Patel et al. in 2015.

\section{CONCLUSION}

Thus, the results of the present study show a high endemicity of MRSA in our hospital in Kolkata. Though our results show $50 \%$ of the MRSA were MDR which could be due to selective antibiotic pressure. But fortunately, none of the isolates were XDR or PDR. This poses a serious problem for drug therapy because the treatment options become limited to potentially toxic antimicrobials like vancomycin, teicoplanin, streptogramins, linezolid and ceftaroline leading to increased mortality and morbidity.

\section{KEY WORDS}

Prevalence, Staphylococcus aureus, MRSA, Pus, Hospital Acquired.

HOW TO CITE THIS ARTICLE: Bhattacharyya I, Banerjee D. The terrorist still lurks in the hospital- prevalence of MRSA. J. Evolution Med. Dent. Sci. 2018;7(52):5509-5512, DOI: 10.14260/jemds/2018/1219

\section{BACKGROUND}

Staphylococcus aureus, a pluripotent pathogen has been the subject of immense interest over the past several decades. Though a part of the normal flora, it is considered as one of the most important human pathogens in community as well as hospital settings worldwide.[1] Community-acquired infections affect the skin and soft tissues, bones and joints, urinary tract and lungs while nosocomial or hospitalacquired infections include surgical wound infections, bacteraemia associated with intravenous devices and other types of prosthetic material and septicaemia.[2] Methicillin resistant Staphylococcus aureus (MRSA) strains, initially described in 1961, has emerged as a multidrug resistant bug worldwide with a prevalence ranging from $4.6 \%$ to $54.4 \% .^{[3,4]}$ The frequency of MRSA has great geographic variation. Most American hospitals report MRSA rates of 5 to $25 \%$, but an increasing number of hospital outbreaks are being reported from USA, Europe, Australia and Japan. ${ }^{[5]}$ The hospitals in south and north India have reported an MRSA prevalence as high as $55 \% .[6,7]$

'Financial or Other Competing Interest': None.

Submission 17-11-2018, Peer Review 11-12-2018,

Acceptance 17-12-2018, Published 24-12-2018.

Corresponding Author:

Debarati Banerjee,

Sugam Sudhir, Block 5,

Flat 6A, 444 (S) Kumrakhali,

Mahamayatala, Kolkata-700103,

West Bengal, India.

E-mail: debarati.ban@gmail.com

DOI: $10.14260 /$ jemds/2018/1219

\section{(c) $(\mathbf{P}) \Theta$}

Intrinsic resistance to all $\beta$-lactam antibiotics and cephalosporins due to low affinity is an important feature of MRSA. Even vancomycin, the drug of choice for severe MRSA infections, has become intermediate sensitive or resistant. Emergence of Vancomycin Intermediate S.aureus (VISA) and Vancomycin resistant S.aureus (VRSA) has now become a global issue including India. ${ }^{[8,9]}$ Also several studies have reported the resistance to the newer antimicrobial agents like linezolid, teicoplanin and daptomycin. The fear of pan drugresistance as cautioned in Gram-negative pathogens cannot be ruled out in S. aureus and our return to a post-antibiotic era.[10] Being a frequent colonizer of the skin, S. aureus predominates in localized suppurative wound and soft tissue infections and since the prevalence and sensitivity pattern of MRSA varies widely geographically, there is an increasing need of antibiotic susceptibility surveillance. The aim of this study is to know the prevalence and drug resistance pattern of MRSA among various pus samples and to know the number of multidrug resistant (MDR) and extensively drug resistant (XDR) strains in order to utilize the information obtained for choosing empirical therapy, to screen colonized patients or staff and formulate appropriate control measures.

\section{MATERIALS AND METHODS}

An observational cross-sectional study was undertaken for one year at the Department of Microbiology, R. G. Kar Medical College and hospital- a tertiary care hospital. The prospective analysis included 226 culture positive pus samples obtained from various anatomical sites of patients admitted at different wards. 
Samples were obtained using sterile cotton tipped swabs from all skin wounds and direct aspiration of pus from deep seated wounds. Wound swabs and catheter tips were transported in brain heart infusion broth. The samples were delivered to the microbiology laboratory within 30 minutes of collection.

All clinical pus samples after reaching the Microbiology department, were immediately inoculated in Nutrient agar, MacConkey agar, and Blood agar and were incubated for 24 hours at $37^{\circ} \mathrm{C}$. Sterile precautions were taken while processing samples. A direct gram stained smear was done and observed for any gram-positive cocci singly or in clusters. After 24 hours, Staphylococcus aureus was identified from samples by colony characteristics such as golden yellow appearance on nutrient agar, pale pink, lactose fermenting colonies on MacConkey agar and yellowish-white butyrous colonies with narrow zone of haemolysis on blood agar. The colonies were sub cultured on selective media like mannitol salt agar which showed yellow coloured colonies indicating mannitol fermentation and phenolphthalein diphosphate agar where the colonies turned pink on exposure to ammonia. Characteristic features such as catalase positivity, slide and tube coagulase positivity, mannitol fermentation, urease production, and VP test positivity were demonstrated to confirm Staphylococcus aureus.

All Staphylococcal strains were tested for susceptibility to Amoxicillin, Azithromycin, Clindamycin, Cefuroxime, Ciprofloxacin, Cotrimoxazole, Doxycycline, Gentamycin, Linezolid and Vancomycin by standard disc diffusion method recommended by the CLSI.[9] Detection of MRSA was done by Kirby Bauer disc diffusion method using Cefoxitin disc (30 $\mu \mathrm{g})$ and incubated at $37{ }^{\circ} \mathrm{C}$ for $16-18$ hours and zones of inhibition were matched with CLSI. Data entered into excel sheet, analysed and tabulated.

\section{RESULTS}

Total number of culture positive pus sample screened were 226 during one-year period. Staphylococcus aureus was the most common cause of surgical and non-surgical wound infections accounting for 102(45.1\%) and Methicillin resistance was documented in 36 (35.3\%) of the S.aureus isolates as shown in Fig. 1. Majority of the MRSA isolates were from males $55.5 \%$. Out of 36 MRSA, most of the isolates $(36.1 \%)$ were observed in the age group of 20-45 years. In relation to ward distribution, maximum number of MRSA were isolated from general surgery ward $(38.9 \%)$ followed by orthopaedics (27.8\%) as shown in Fig. 2.

Maximum susceptibility was seen to vancomycin (100\%) and linezolid (100\%) followed by levofloxacin (61\%), doxycycline (58\%) and chloramphenicol (58\%). Macrolides $(11 \%)$, cotrimoxazole $(14 \%)$ was found to be less sensitive. None of the isolates were found to be sensitive to amoxicillin. According to the definition of multidrug resistance as acquired non-susceptibility to at least one agent in three or more antimicrobial categories, $18(50 \%)$ were found to be multidrug resistant (MDR). None of the isolates were found to be extensively- drug resistant (XDR) or pan-drug resistant (PDR).

\section{Distribution of MRSA and MSSA among isolates collected $(\mathrm{n}=102)$}

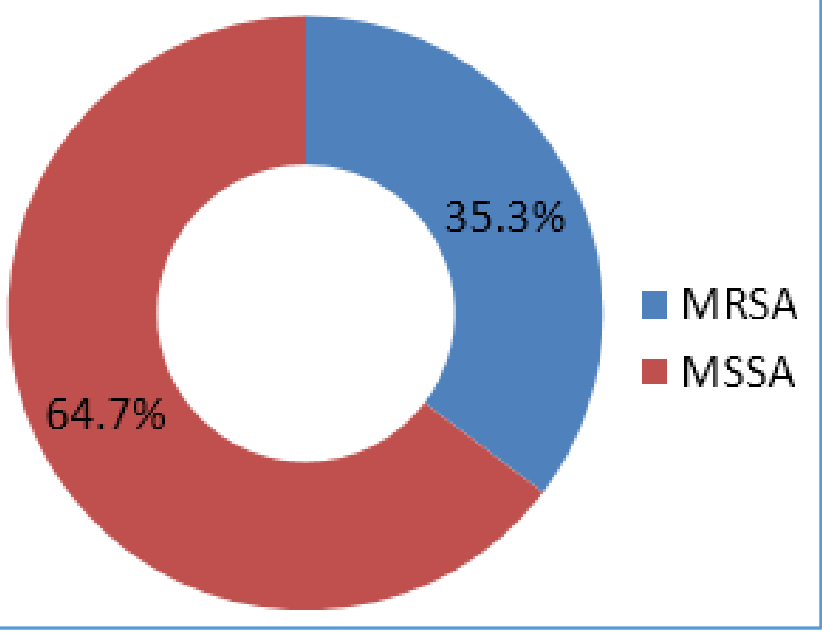

Figure 1

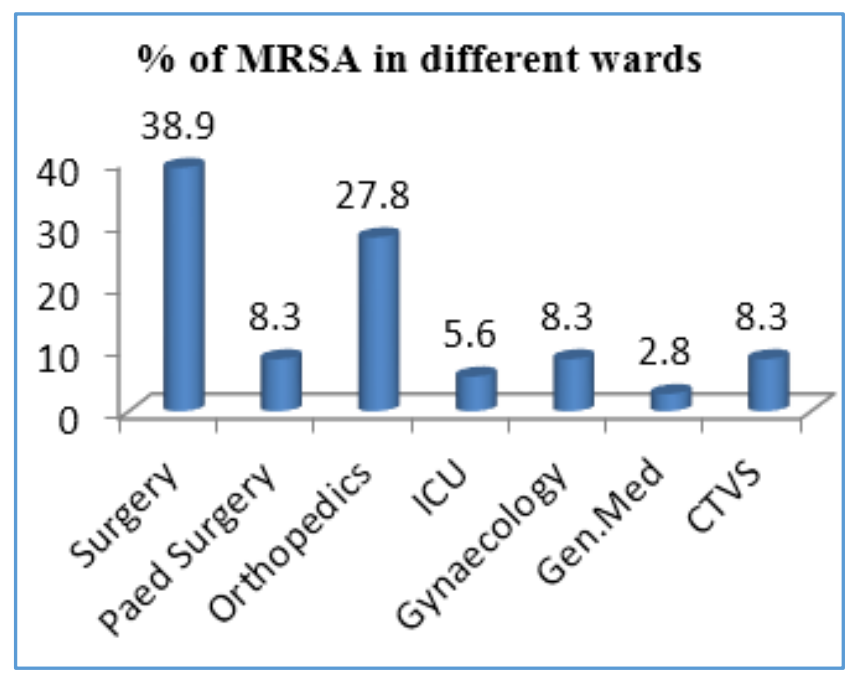

Figure 2

\section{Susceptibility pattern of MRSA isolates}

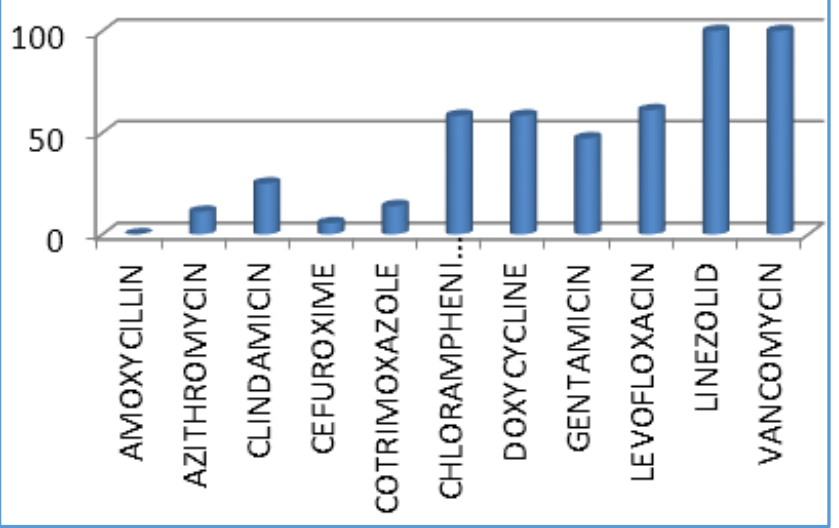

Figure 3 


\section{DISCUSSION}

MRSA, an organism well known for its multidrug resistance, varies according to the geographical pattern. In the present study from Central Kolkata, the prevalence of MRSA was found to be $35.5 \%$ which was significantly higher than a study (28.8\%) done by Uma Devi[1] in Andhra Pradesh, but at par with a study done by Sangram S Patel et al.[11] A study by Arti Tyagi et al on 2080 pus samples at AIIMS, New Delhi, documented an even higher MRSA prevalence of $44 \%$. Prevalence of MRSA varies in different countries. Over $50 \%$ prevalence rate of MRSA was reported in Portugal and Italy; $25 \%$ in England, Greece and France; $2 \%$ in the Netherlands and Switzerland.[12] Prevalence of MRSA ranged from $23.6 \%$ in Australia to over $61 \%$ in Taiwan and Singapore, and more than $70 \%$ in Japan and Hong Kong.[13]

The usual inclination of MRSA infection is generally seen in the intensive care unit followed by medicine and surgery wards, where most patients required higher generation, toxic antibiotics. Surprisingly in this study highest occurrence of MRSA was observed in surgical wound infection in General surgery followed by orthopaedic wards. This is mostly because of cross infection due to overcrowding of patients, workload and understaffing.

In the present study, all the isolates of MRSA were susceptible to vancomycin and linezolid which is comparable to studies by Rajaduraipandi et al. and Rao et al.[14,15] However S.aureus isolates with reduced susceptibility to vancomycin was reported earlier by CDC and with complete resistance to vancomycin was reported by Japanese workers.[16,17] In the recent years Tiwari et al. in a study from northern part of India in 2006 found, out of 381 MRSA isolates, 2 were vancomycin resistant while 6 were intermediately resistant to vancomycin.[18] Hence vancomycin should always be used as a reserve drug and can be used as empirical therapy for life threatening MRSA infections but step-down therapy to susceptible antibiotics should be started as soon as antibiotic susceptibility data is available.

\section{CONCLUSION}

Thus, the results of the present study show a high endemicity of MRSA in our hospital in Kolkata. Though our results show $50 \%$ of the MRSA were MDR which could be due to selective antibiotic pressure, but to the relief that none of the isolates were XDR or PDR. This poses a serious problem for drug therapy because the treatment options become limited to potentially toxic antimicrobials like vancomycin, teicoplanin, streptogramins, linezolid and ceftaroline leading to increased mortality and morbidity. Hence preventing colonization and infection of patients on one hand and screening and identifying MRSA carriers on the other hand in the high-risk wards remains the most effective way to control the spread of MRSA. Simple measures like patient isolation, cohorting doctors and nurses working with MRSA infected patients, routine screening of health care workers for MRSA carriage by sending nasal swabs and nail bed samples to microbiology laboratory, proper treatment of MRSA carriers for eradication, strict enforcement of hand washing and early discharge of infected patients will go a long way towards reducing the spread of this deadly bug in this hospital. Also, indiscriminate use of antibiotics should be stopped and routinely all microbiological samples should be sent for in vitro susceptibility. This will help in detection of multidrug resistant pathogens and start appropriate treatment at the earliest. Thus, in future, based on this study, antibiotic policies should be made and implemented by setting up and/or strengthening hospital infection control committee. Antimicrobial stewardship programs can be implemented to reduce inappropriate use of antimicrobials, thereby controlling the development of resistance. Though this is a limitation of this study due to unavailability of resources, genetic study of mecA and mecC genes can be done for epidemiological analysis of MRSA strains in different wards of the hospital.

\section{REFERENCES}

[1] Umadevi S. Methicillin resistant staphylococcus aureus in pus samples. Int J Med Science and Clin Invention 2017;4(12):3374-6.

[2] Kaur H, Purwar S, Saini A, et al. Status of methicillinresistant Staphylococcus aureus infections and evaluation of PVL producing strains in Belgaum, South India. JKIMSU 2012;1(2):43-51.

[3] Swanston WH. Methicillin resistant Staphylococcus aureus. West Indian Med J 1999;48(1):20-2.

[4] Kac G, Buu-Hoi A, Herrison E, et al. Methicillin resistant staphylococcus aureus. Nosocomial acquisition and carrier state in a wound care center. Arch Dermatol 2000;136(6):735-9.

[5] Ayliffe GA, Duckworth GJ, Brumfitt W, et al. Guidelines for the control of epidemic methicillin-resistant Staphylococcus aureus. Report of a combined working party of the Hospital Infection Society and British Society for Antimicrobial Chemotherapy. J Hasp Infect 1986;7(2):193-201.

[6] Khadri H, Alzohairy M. Prevalence and antibiotic susceptibiliity pattern of Methicillin-resistance and coagulase-negative Staphylococci in a tertiary hospital in India. International Journal of Medicine and Medical Sciences 2010;2(4):116-20.

[7] Anupurba S, Sen MR, Nath G, et al. Prevalence of MRSA in a tertiary care referral hospital in Eastern UP. Indian J Medical Microbiol 2003;21(1):49-51.

[8] Menezes GA, Harish BN, Sujatha S, et al. Emergence of vancomycin - intermediate Staphylococcus species in Southern India. Journal of Medical Microbiology 2008;57(Pt 7):911-2.

[9] CLSI. Performance Standards for Antimicrobial Susceptibility Testing: Twenty second informational supplement. CLSI document M100-S25. Wayne, PA: Clinical and Laboratory Standards Institute, 2015.

[10] Kaur DC, Chate SS. Study of antibiotic resistance pattern in methicillin resistant staphylococcus aureus with special reference to newer antibiotic. J Glob Infect Dis 2015;7(2):78-84.

[11] Patel SS, Sengupta BC, Venkatesan A. Incidence of Methicillin Resistant Staphylococcus aureus [MRSA] in pus samples and associated risk factors in a tertiary care hospital. Int J Adv Res 2015;3(5):1097-102.

[12] Orrett FA. Antimicrobial resistance in Trinidad: hospital practice strains verses community practice strains of Staphylococcus aureus. Jpn J Infect Dis 1997;25:663-6. 
[13] Diekema DJ, Pfaller MA, Schmitz FJ, et al. Survey of infections due to Staphylococcus species: frequency of occurrence and antimicrobial susceptibility of isolates collected in the United States, Canada, Latin America, Europe and the Western Pacific region for the SENTRY Antimicrobial Surveillance Program, 1997-1999. Clin Infect Dis 2001;32(Suppl 2):S114-S32.

[14] Rajaduraipindi K, Mani KR, Panneerselvam K, et al. Prevalence and antimicrobial susceptibility pattern of MRSA - a multicentre study. Ind J Med Microbiol 2006;24 (1):34-8.

[15] Rao Bandaru N, Prabhakar T. Prevalence and antimicrobial susceptibility pattern of MRSA at Vishakhapatnam. J Pathol \& BioMed Sciences 2011;4 (03):1-5.
[16] Centres for Disease Control. Staphylococcus aureus with reduced susceptibility to Vancomycin - United States. MMWR 1997;46:765-88.

[17] Hiramatsu K, Aritaka N, Hanaki H, et al. Dissemination in Japanese hospitals of strains of Staphylococcus aureus heterogeneously resistant to vancomycin. Lancet 1997;350(9092):1670-3.

[18] Tiwari HK, Sen MR. Emergence of vancomycin resistant Staphylococcus aureus (VRSA) from a tertiary care hospital from northern part of India. BMC Infect Dis 2006;6:156. 